\title{
Current drug use and lack of HIV virologic suppression: point-of-care urine drug screen versus self-report
}

\author{
Han-Zhu Qian ${ }^{1,2^{*}}$, Valerie J Mitchell ${ }^{3}$, Sally Bebawy ${ }^{3,4}$, Holly Cassell ${ }^{1}$, Gina Perez ${ }^{3}$, Catherine C McGowan ${ }^{3,4}$, \\ Timothy R Sterling ${ }^{3,4}$, Sten H Vermund ${ }^{1}$, Richard D'Aquila ${ }^{5}$ and Todd Hulgan ${ }^{3,4}$
}

\begin{abstract}
Background: There have been inconsistent findings on the association between current drug use and HIV disease progression and virologic suppression. Drug use was often measured using self-report of historical use. Objective measurement of current drug use is preferred.

Methods: In this cross-sectional study, we assessed drug use through Computer-Assisted Self Interviews (CASI) and point-of-care urine drug screen (UDS) among $225 \mathrm{HIV}$-infected patients, and evaluated the association between current drug use and virologic suppression.

Results: About half (54\%) of participants had a positive UDS, with a lower self-reported rate by CASI (42\%) (Kappa score $=0.59$ ). By UDS, 36.0\% were positive for marijuana, $25.8 \%$ for cocaine, $7.6 \%$ for opiates, and $2.2 \%$ for methamphetamine or amphetamine. Factors associated with virologic suppression (plasma HIV RNA $<50$ copies $/ \mathrm{mL}$ ) were Caucasian race $(P=0.03)$, higher CD4 count $(P<0.01)$, current use of antiretroviral therapy (ART) $(P<0.01)$, and a negative UDS $(P<0.01)$. Among 178 current ART users, a positive UDS remained significantly associated with lower likelihood of virologic suppression $(P=0.04)$.

Conclusions: UDS had good agreement with CASI in detecting frequently used drugs such as marijuana and cocaine. UDS at routine clinic visits may provide "real-time" prognostic information to optimize management.
\end{abstract}

Keywords: Drug use, HIV, Computer-assisted self-interview, Urine drug screen, Antiretroviral therapy, Virologic suppression

\section{Background}

The introduction of antiretroviral therapy (ART) has resulted in a decline in AIDS-defining events and mortality in HIV-infected populations. Studies suggest, however, that people who use drugs (PWUD), particularly those who inject drugs, may not achieve the same benefits from treatment as persons who do not use drugs [1,2]. Even when controlled for adherence to ART, outcomes for PWUD may be poorer compared to non-users [3]. However, other studies have shown conflicting results with minimal or no association between drug use and HIV

\footnotetext{
*Correspondence: han-zhu.qian@vanderbilt.edu

${ }^{1}$ Vanderbilt Institute for Global Health, Vanderbilt University, Nashville,

Tennessee, USA

${ }^{2}$ Division of Epidemiology, Department of Medicine, Vanderbilt University,

Nashville, Tennessee, USA

Full list of author information is available at the end of the article
}

disease progression [4,5]. These discordant findings may be due to differences in measuring drug use, such as the type of drugs used, route of administration, dose and frequency, current or past use, and polydrug use $[4,6]$.

There are numerous ways of measuring drug use in research and clinical care settings. Traditional paper-based questionnaire surveys, either self-completed or administered by an interviewer, are relatively inexpensive and easy to implement, but are subject to social desirability bias due to stigma associated with drug using behaviors $[7,8]$. Computer Assisted Self Interview (CASI) may reduce but not eliminate such bias [9-11]. Both questionnaire and CASI interviews can assess past and current use of drugs. In contrast, direct drug testing by urine drug screen (UDS) detects metabolites in urine samples with good sensitivity and specificity [12,13], and therefore 
provides an objective assessment, but is more costly and may be impractical for routine use in some settings. As the window for detecting metabolites in urine typically ranges from a few hours to two weeks, a positive UDS indicates current or recent drug use [13]. Studies on the relationship between use of illicit drugs and HIV disease outcomes often use self-reported history of drug use. There is a scarcity of literature on validation of reported drug use and on the assessments of current drug use and its potential association with HIV treatment outcomes among HIV-infected patients [3,14]. We assessed the agreement between CASI and UDS results in this group of HIV-infected participants, and the relationship between virologic suppression and drug use as assessed by UDS.

\section{Methods}

\section{Study setting and target population}

The study was conducted during June 2010 and December 2011 at the Vanderbilt Comprehensive Care Clinic (VCCC), a large outpatient HIV clinic in Nashville, Tennessee that provides integrated HIV and psychiatric care, and social work services to over 2,800 patients in active care. An estimated $80 \%$ of patients at VCCC are from Middle Tennessee; the remaining are from other parts of Tennessee or from surrounding states of Kentucky, Georgia, West Virginia, Alabama and Arkansas. Patients were recruited by a research nurse during clinic visits at VCCC, where they read brochures about this drug userelated study then voluntarily decided to participate in the study. About one out of three patients approached by the nurse agreed to participate in the study. Each participant provided a written informed consent for completing a CASI interview, providing urine sample for UDS, and giving permission of use of their clinical data. A $\$ 10$ gift card was given to participants who completed the study procedures. The study protocol was approved by the institutional review board of Vanderbilt University. Approval was granted to incorporate data collected from this study into the VCCC data and specimen repository for future research use, but study data were kept separate from the medical record.

\section{Computer-assisted self interview}

CASI was conducted to collect data on drug and alcohol use, depression, and adherence to antiretroviral therapy (ART). Participants were asked 3-item AUDIT alcohol consumption questions (AUDIT-C) to query for problematic drinking $[15,16]$. Participants were also asked to indicate whether or not they had used a variety of illicit substances in the past 7 days, 6 months and lifetime using a modified version of WHO-ASSIST (Alcohol, Smoking \& Substance Involvement Screening Test) questionnaire. Substances included cocaine/crack, marijuana, heroin or other opiates, methamphetamine (crystal meth), pain medications, benzodiazepines, and inhalants. The 9item Patient Health Questionnaire (PHQ-9) Depression Module was used to screen for depression. The 4-item AIDS Clinical Trials Group (ACTG) Questionnaire for Adherence to Antiretroviral Medications was used to assess ART adherence [17].

\section{Urine drug screen}

All participants undertook a point-of-care UDS. The FDA-approved QuickScreen ${ }^{\text {mi }}$ Pro 5 Drug Test Card (Craig Medical Distribution, Inc. Vista, CA) was used [18]. It is a one-step panel immunoassay for the qualitative detection of amphetamine, benzoylecgonine (cocaine metabolite), methamphetamine (including Ecstasy), opiates (morphine/ heroin metabolite) and marijuana in human urine. Amphetamine has a half-life of 4-24 hours in the body and methamphetamine has a half-life of 9-24 hours; cocaine can generally be detected for 24-48 hours after exposure; the half-life of opiate drugs may range from 2 to 32 hours, e.g., 8-12 hours for heroin; marijuana remains detectable for 3-10 days after smoking. The visual one-step panel urine test provides a qualitative "yes or no" report in $5 \mathrm{mi}$ nutes. According to the package inserts of the testing kit, the agreement rates of detecting the individual drugs using the QuickScreen ${ }^{\text {Tx }}$ Pro 5 Drug Test Card and confirmatory gas chromatography-mass spectrometry range from 95\% to $98 \%$. A trained research nurse performed UDS and recorded the results. While waiting for UDS results, patients completed CASI.

\section{Clinical data}

Data retrieved from the clinical database at VCCC included socio-demographics, ART use, and the latest values of CD4 count and HIV RNA in plasma measured by standard procedures as part of routine care. The values of CD4 and viral load that were closest to the survey date were used in the analysis, most within 3 months of the survey and few between 3-6 months. Virologic suppression was defined as plasma HIV RNA $<50$ copies $/ \mathrm{mL}$.

\section{Data analysis}

The AUDIT-C is scored on a scale of 0 to 12 , and the scores of $\geq 4$ in men and $\geq 3$ in women were defined as unhealthy alcohol use $[15,19]$. Current drug use was defined as a positive result on any of five tested drugs in UDS. We did not assess relationships with specific drugs due to limited study sample size. The PHQ-9 is scored on a scale of 0 to 27 , and the scores of $\geq 10$ were defined as major depression (mediate or severe depression) [20]. ART adherence was defined by whether having missed at least one dose of ART in past 4 days. Virologic suppression was defined as HIV-1 RNA $<50$ copies $/ \mathrm{mL}$.

We calculated the detection rate of drug use by UDS and the rates of drug use during the past 7 days, the past 
6 months, and lifetime use by CASI self-report. We then calculated the agreement (Kappa score) of detecting current drug use by CASI 7-day report and UDS. We considered self-reported 7-day drug use as current use. The Kappa scores are interpreted as: < 0 indicating no agreement, $0-0.20$ slight, $0.21-0.40$ fair, $0.41-0.60$ moderate, $0.61-0.80$ substantial, and $0.81-1$ almost perfect agreement [21].

We performed multivariate logistic regression analysis in the entire study sample to evaluate the relationship between current use of any drugs as determined by UDS and virologic suppression while adjusting for covariates. We also performed subgroup analysis among patients who had received ART only, because prescription of ART may be less likely to occur for drug using patients than for non-users [22]. We selected covariates and fitted regression models based on our cohort analysis in the same study population [1]. We re-analyzed the data and did not find interaction between ART and drug use. Therefore, we use the parsimonious models without interaction term.

\section{Results}

A total of $226 \mathrm{HIV}$-infected patients participated in the study; one participant did not provide a urine sample for UDS and was excluded from the analysis. Of 225 participants, about 30\% were female; the mean age was 43 years (median 49; range 19-66); 38\% were Caucasians, and 59\% were African Americans (Table 1).

Nearly $38 \%$ of participants reported unhealthy alcohol use; $25 \%$ had moderate or severe depression (Table 1). Of the $80 \%$ of patients who were prescribed ART, $32 \%$ reported missing at least one dose in the past 4 days (data not shown).

Table 2 presents the prevalence of drug use by UDS and CASI. About half (53.5\%) had a positive UDS for any drug. Over one third (36.0\%) were positive for marijuana, 25.8\% for cocaine, $7.6 \%$ of opiates, $2.2 \%$ for methamphetamine or amphetamine. The CASI reported rate of using any drugs in the past 7 days was $42.0 \%$, which was significantly lower than that by UDS (less than the lower bound of 95\% confidence interval of UDS rate). CASI also reported lower rates of marijuana (28.6\%) and cocaine (18.9\%) use than corresponding positive UDS rates. Ninety-two percent of participants reported any lifetime drug use. Though nearly a quarter of participants reported ever injecting drugs, very few reported injection drug use in the past seven days $(0.4 \%)$ or six months (1.8\%).

Table 3 presents the agreement statistic Kappa values for detecting drug use by UDS and CASI reported 7-day drug use. The Kappa values for marijuana and cocaine were 0.68 and 0.71 , respectively, indicating substantial agreement; and the Kappa value for any drugs was 0.59, indicating moderate agreement. For other infrequently used drugs, the agreement was poor.

Factors associated with virologic suppression were Caucasian race (versus non-Caucasian; adjusted odds ratio $[\mathrm{AOR}] 2.5 ; 95 \%$ confidence interval [CI] 1.1-5.7), CD4 count (per 10 cell/uL increase; AOR 1.003; 95\% CI 1.001-1.004), current use of ART (AOR 8.3; 95\% CI 3.122.3), and current drug use by UDS (a positive UDS was associated with a significantly lower odds of virologic suppression: AOR 0.3; 95\% CI 0.1-0.7). There was still a statistically significant association between current drug use and lack of virologic suppression among the subgroup of participants who were currently prescribed ART (AOR 0.4; 95\% CI 0.2-0.9) (Table 4).

\section{Discussion}

Our study is one of few studies comparing self-report and UDS in measuring drug use [14,23-25] and the only study to our knowledge among HIV-infected patients. We found that CASI and point-of-care UDS had fair agreement in detecting current use of common drugs such as marijuana and cocaine among HIV-infected patients, but had poor agreement for infrequently used

Table 1 Description of study participants by UDS status

\begin{tabular}{|c|c|c|c|c|}
\hline Variable & $\begin{array}{c}\text { Total sample } \\
(\mathrm{N}=225)\end{array}$ & $\begin{array}{l}\text { UDS positive } \\
(\mathrm{N}=121)\end{array}$ & $\begin{array}{l}\text { UDS negative } \\
(\mathrm{N}=104)\end{array}$ & $P$-value \\
\hline Sex (male) & $157(69.8 \%)$ & $86(54.8 \%)$ & $71(45.2 \%)$ & 0.65 \\
\hline Age, year (mean $\pm S D$ ) & $42.7 \pm 10.1$ & $44.0 \pm 10.0$ & $41.2 \pm 10.2$ & 0.04 \\
\hline Race & & & & 0.01 \\
\hline White & $86(38.2 \%)$ & $40(46.5 \%)$ & $46(53.5 \%)$ & \\
\hline Black & $132(58.7 \%)$ & $80(60.6 \%)$ & $52(39.4 \%)$ & \\
\hline Other & $7(3.1 \%)$ & $1(14.3 \%)$ & $6(85.7 \%)$ & \\
\hline Unhealthy alcohol use & $85(37.7 \%)$ & $49(57.6 \%)$ & $36(42.4 \%)$ & 0.71 \\
\hline Major depression ( $\geq$ moderate level) & $57(25.3 \%)$ & $29(50.9 \%)$ & $28(49.1 \%)$ & 0.67 \\
\hline ART, yes & $179(79.6 \%)$ & $94(52.5 \%)$ & $85(47.5 \%)$ & 0.63 \\
\hline
\end{tabular}

Note: UDS: urine drug screen; SD: standard deviation. 
Table 2 Prevalence of drug use by point-of-care urine drug screen (UDS) and Computer-Assisted Self Interview (CASI) among 225 HIV-infected patients in middle Tennessee, USA (\%)

\begin{tabular}{ccccc}
\hline Drug & $\begin{array}{c}\text { UDS } \\
\text { proportion } \\
(\mathbf{9 5 \% ~ C l ) *}\end{array}$ & $\begin{array}{c}\text { Past } \\
\text { 7 days }\end{array}$ & $\begin{array}{c}\text { Past } \\
\mathbf{6} \text { months }\end{array}$ & Lifetime \\
\hline Any drugs & $53.5(47.0-60.0)$ & $42.0^{\ddagger}$ & 59.3 & 92.4 \\
Marijuana & $36.0(29.7-42.3)$ & $28.6^{\ddagger}$ & 44.1 & 85.0 \\
Cocaine & $25.8(20.1-31.5)$ & $18.9^{\ddagger}$ & 29.5 & 66.8 \\
Opiates & $7.6(4.1-10.1)$ & 7.5 & 18.5 & 43.8 \\
Meth/amphetamine & $2.2(0.3-4.1)$ & 1.8 & 6.2 & 25.2 \\
Injection & N/A & 0.4 & 1.8 & 23.7 \\
\hline
\end{tabular}

Note: $\mathrm{Cl}$ : confidence interval; N/A: not applicable.

*95\% Cl was calculated in order to assess whether or not the CASI point estimate was likely to be within $95 \% \mathrm{Cl}$ of the UDS estimate.

${ }^{\mp}$ The prevalence of 7-day drug use by CASI is less than the lower bound of $95 \% \mathrm{Cl}$ of the prevalence by UDS.

drugs. CASI tends to underreport current drug use compared to UDS. Only about $10 \%$ of participants $(n=24)$ ever received drug or alcohol treatment in the past 6 months, and the treatment experience had no significant impact on the difference of detecting drug use by UDS and self-report. A study among college students showed that the overall result in detecting any drug use was satisfactory, while the sensitivity of self-report on more stigmatized drugs such as cocaine was lower than that on less stigmatized drugs such as marijuana (cannabis) among college students [14], and these findings are consistent with another study among men who have sex with men and the general population [24]. One advantage of CASI is that it can assess drug use for longer periods of time, such as past 6 months or lifetime. However, serial UDS at repeated clinic visits may also allow longer-term assessment and is also less likely to be subject to reporting bias.

Lifetime and current drug use was very common among this group of HIV-infected, predominantly ARTtreated participants recruited during clinical visits and thus engaged in care to some degree. Current drug use was associated with lower likelihood of virologic suppression among ART-treated participants. Drug use may lead to suboptimal HIV treatment outcomes through both biological and behavioral mechanisms. For example, PWUD may have poor adherence to ART; illicit drugs such as amphetamines, cocaine, marijuana, and opiates may alter immune function and increase susceptibility to infection $[4,26]$. Rapid point-of-care screening for drug use at routine clinic visits may provide useful "real time" prognostic information for HIV treatment efficacy and optimize management decisions by identifying persons at greatest risk for treatment failure, guiding decisions regarding targeted adherence counseling, timely referral to appropriate drug use treatment programs, and testing for viral resistance. We did not find an association between unhealthy alcohol use and virologic suppression, although there is biological plausibility [27]. Evidences on the relationship between heavy alcohol consumption and HIV outcomes are inconsistent [27].

Table 3 Agreement in detecting drug use by point-of-care urine drug screen (UDS) and Computer-Assisted Self Interview (CASI)

\begin{tabular}{|c|c|c|c|c|c|}
\hline \multicolumn{2}{|c|}{ CASI reported 7-day use } & \multicolumn{3}{|c|}{ UDS } & \multirow[t]{2}{*}{ Kappa coefficient } \\
\hline & & $(+)$ & $(-)$ & total & \\
\hline \multirow[t]{3}{*}{ Any drugs } & $(+)$ & $84(37.3 \%)$ & $10(4.4 \%)$ & $94(41.8 \%)$ & 0.59 \\
\hline & $(-)$ & $37(16.4 \%)$ & $94(41.8 \%)$ & $131(58.2 \%)$ & \\
\hline & Total & $121(53.8 \%)$ & $104(46.2 \%)$ & $225(100.0 \%)$ & \\
\hline \multirow[t]{3}{*}{ Marijuana } & $(+)$ & $57(25.3 \%)$ & $8(3.6 \%)$ & $65(28.9 \%)$ & 0.68 \\
\hline & $(-)$ & $24(10.7 \%)$ & $136(60.4 \%)$ & 160 (71.1\%) & \\
\hline & Total & $81(36.0 \%)$ & $144(64.0 \%)$ & $225(100.0 \%)$ & \\
\hline \multirow[t]{3}{*}{ Cocaine } & $(+)$ & $39(17.3 \%)$ & $3(1.3 \%)$ & $42(18.7 \%)$ & 0.71 \\
\hline & $(-)$ & $19(8.4 \%)$ & $164(72.9 \%)$ & $183(81.3 \%)$ & \\
\hline & Total & $58(25.8 \%)$ & $167(74.2 \%)$ & $225(100.0 \%)$ & \\
\hline \multirow[t]{3}{*}{ Opiates } & $(+)$ & $2(0.9 \%)$ & $15(6.7 \%)$ & $17(7.6 \%)$ & 0.05 \\
\hline & $(-)$ & $15(6.7 \%)$ & $193(85.8 \%)$ & 208 (92.4\%) & \\
\hline & Total & $17(7.6 \%)$ & $208(92.4 \%)$ & $225(100.0 \%)$ & \\
\hline \multirow[t]{3}{*}{ Methamphetamine } & $(+)$ & $0(0.0 \%)$ & $4(1.8 \%)$ & $4(1.8 \%)$ & -0.01 \\
\hline & $(-)$ & $5(2.2 \%)$ & $216(96.0 \%)$ & $221(98.2 \%)$ & \\
\hline & Total & $5(2.2 \%)$ & $220(97.8 \%)$ & 225 (100.0\%) & \\
\hline
\end{tabular}


Table 4 Multivariate logistic regression analyses of factors associated with HIV suppression (<50 copies/mL) among 225 HIV-infected patients

\begin{tabular}{|c|c|c|c|c|c|c|}
\hline \multirow{3}{*}{ Variable } & \multicolumn{3}{|c|}{ Model 1: overall } & \multicolumn{3}{|c|}{ Model 2: ART users } \\
\hline & \multicolumn{3}{|c|}{$(\mathrm{N}=225)$} & \multicolumn{3}{|c|}{$(\mathrm{N}=178)$} \\
\hline & Adjusted OR & $95 \% \mathrm{Cl}$ & $P$-value & Adjusted OR & $95 \% \mathrm{Cl}$ & $P$-value \\
\hline Sex (male vs. female) & 1.38 & $0.63-3.02$ & 0.42 & 1.29 & $0.53-3.18$ & 0.57 \\
\hline Age, 1 year increase & 1.01 & $0.97-1.05$ & 0.54 & 1.02 & $0.97-1.07$ & 0.33 \\
\hline Race (Caucasian vs. non-Caucasian) & 2.50 & $1.10-5.68$ & 0.03 & 3.29 & $1.20-9.06$ & 0.02 \\
\hline CD4 count, 10 cells/uL increase & 1.003 & $1.001-1.004$ & $<0.01$ & 1.003 & $1.001-1.004$ & $<0.01$ \\
\hline Current use of ART & 8.29 & $3.08-22.30$ & $<0.01$ & & & \\
\hline Missed at least one dose of ART in past 4 days & & & & 0.93 & $0.37-2.35$ & 0.87 \\
\hline Current drug use by UDS & 0.33 & $0.15-0.73$ & $<0.01$ & 0.38 & $0.15-0.94$ & 0.04 \\
\hline Unhealthy alcohol use & 1.12 & $0.52-2.44$ & 0.77 & 1.02 & $0.43-2.44$ & 0.97 \\
\hline Major depression ( $\geq$ moderate level) & 0.72 & $0.31-1.69$ & 0.44 & 0.89 & $0.32-2.43$ & 0.81 \\
\hline
\end{tabular}

OR: odds ratio; $\mathrm{Cl}$ : confidence interval; UDS: urine drug screen.

Our study suggests the potential treatment benefits of integrating routine screening for substance use in HIV clinics, with and without the use of UDS. The detection of drug use will alert physicians to consider providing drug abuse treatment and counseling in order to achieve optimal HIV treatment outcome. Meanwhile, there are numerous issues that may arise from implementing routine point-of-care UDS for all HIV-infected patients. Patients may have concerns about drug use status being disclosed to their employers or insurers, and their views on integrated HIV primary care and drug screening and treatment should be taken into consideration [28]. Physicians may provide differential care to drug-using patients, including deferring ART treatment [29]. The cost of UDS may not be reimbursed from patients' insurance plans. Concerns about routine UDS may increase the likelihood that some patients would miss medical appointments. Therefore, instead of universal UDS for all HIV-infected patients, opt-out UDS could be considered. Finally, from the perspective of clinic administrators, implementing routine point-of-care UDS would require additional personnel and lab space.

Our study has several limitations. The length of time during which different drugs or their metabolites can be detected in urine samples depends on many factors, including chemical properties (e.g., half-life), metabolism rates and excretion routes, amount consumed, administration route, frequency and chronicity of use, and individual variations in patients' physical health, exercise, diet, weight, gender, and fluid intake that may affect excretion rates [30], and it is generally 2-5 days with a range from 24 hours up to 14 days. The QuickScreen ${ }^{\text {m }}$ Pro 5 Drug Test Card is convenient to use but provides only a preliminary analytical test result; in comparison, confirmatory methods such as gas chromatography/mass spectrometry (GC/MS) are expensive. Therefore, our
UDS findings might underestimate drug use. We did not assess use of prescription opioid use, which might be common among HIV-infected patients [31]. This topic warrants research. The study participants were recruited as a convenience sample through brochures advertising a drug use-related study. This recruitment likely led to an enrichment of the population who currently used drugs and an overestimation of current drug use, but it should not affect the validity of assessing test agreement or the relationship between current drug use and virologic suppression. Adherence to ART, a key covariate in assessing the relationship between drug use and viral suppression, was based on self-report, which may result in overestimation of adherence; pharmacy data and Medication Event Monitoring System (MEMS) could be considered as alternative measures [32,33]. Due to the cross-sectional nature of the study, we could not ascertain the temporal relationship between drug use and virologic suppression. Prospective cohort studies with longitudinal assessment of point-of-care drug use screening and HIV outcomes are needed.

\section{Conclusions}

UDS at routine clinic visits may provide "real-time" prognostic information to optimize HIV care, but studies are needed to evaluate its acceptability among patients and providers.

Competing interests

The authors declare that they have no competing interests.

Authors' contributions

HZQ, SHV: Concept design. VJM, SB, HC: Data acquisition. HZQ, TH: Data analysis; $H Z Q, T R S, R D A$, TH: Data interpretation; HZQ: Drafting and revising; $H C, G P, C C M, T R S, R D A, T H$ : Revising. All authors read and approved the final manuscript. 


\section{Acknowledgements}

This study was supported by Vanderbilt-Meharry Center for AIDS Research Developmental Grant (NIH grant P30 Al 54999) and Vanderbilt Institute for Clinical and Translational Research grant support (1 UL1 RR024975). This project was conducted utilizing the services of the Survey Research Shared Resource at Vanderbilt Medical Center which is supported in whole or in part by the Cancer Center Supporting Grant \# P30CA068485 funded by the National Cancer Institute. The funders had no role in study design, data collection, analysis, decision to publish or preparation of the manuscript.

\section{Author details}

${ }^{1}$ Vanderbilt Institute for Global Health, Vanderbilt University, Nashville, Tennessee, USA. ${ }^{2}$ Division of Epidemiology, Department of Medicine, Vanderbilt University, Nashville, Tennessee, USA. ${ }^{3}$ Division of Infectious Diseases, Department of Medicine, Vanderbilt University, Nashville, Tennessee, USA. "Vanderbilt Comprehensive Care Clinic, Nashville, Tennessee, USA. ${ }^{5}$ Division of Infectious Diseases, Department of Medicine, Northwestern University Feinberg School of Medicine, Chicago, Illinois, USA.

Received: 18 October 2013 Accepted: 15 September 2014 Published: 18 September 2014

\section{References}

1. Qian HZ, Stinnette SE, Rebeiro PF, Kipp A, Shepherd BE, Samenow CP Jenkins CA, McGowan CC, Hulgan T, Sterling TR: The relationship of history injecting and non-injecting drug use with HIV disease progression. J Subst Abuse Treat 2011, 41(1):14-20.

2. Perez-Hoyos S, del Amo J, Muga R, del Romero J, de Garcia OP, Guerrero R, Hernandez-Aguado I: Effectiveness of highly active antiretroviral therapy in Spanish cohorts of HIV seroconverters: differences by transmission category. Aids 2003, 17(3):353-359.

3. Rasbach DA, Desruisseau AJ, Kipp AM, Stinnette S, Kheshti A, Shepherd BE, Sterling TR, Hulgan T, McGowan CC, Qian HZ: Active cocaine use is associated with lack of HIV-1 virologic suppression independent of nonadherence to antiretroviral therapy: use of a rapid screening tool during routine clinic visits. AIDS Care 2013, 25(1):109-117.

4. Kipp AM, Desruisseau AJ, Qian HZ: Non-injection drug use and HIV disease progression in the era of combination antiretroviral therapy. J Subst Abus Treat 2011, 40(4):386-396.

5. Chaisson RE, Keruly JC, Moore RD: Race, sex, drug use, and progression of human immunodeficiency virus disease. N Engl J Med 1995, 333(12):751-756.

6. Kapadia F, Cook JA, Cohen MH, Sohler N, Kovacs A, Greenblatt RM, Choudhary I, Vlahov D: The relationship between non-injection drug use behaviors on progression to AIDS and death in a cohort of HIV seropositive women in the era of highly active antiretroviral therapy use. Addiction 2005, 100(7):990-1002

7. Johnson T, Fendrich M: Modeling sources of self-report bias in a survey of drug use epidemiology. Ann Epidemiol 2005, 15(5):381-389.

8. Sloan JJ, Bodapati MR, Tucker TA: Respondent misreporting of drug use in selfreports: social desirability and other correlates. J Drug Issues 2004, 34:269.

9. Gribble JN, Miller HG, Rogers SM, Turner CF: Interview mode and measurement of sexual behaviors: methodological issues. J Sex Res 1999, 36(1):16-24.

10. Hutton HE, Lyketsos CG, Zenilman JM, Thompson RE, Erbelding EJ: Depression and HIV risk behaviors among patients in a sexually transmitted disease clinic. Am J Psychiatry 2004, 161(5):912-914.

11. Islam MM, Topp L, Conigrave KM, van Beek I, Maher L, White A, Rodgers C, Day CA: The reliability of sensitive information provided by injecting drug users in a clinical setting: clinician-administered versus audio computer-assisted self-interviewing (ACASI). AIDS Care 2012, 24(12):1496-1503.

12. Holler JM, Bosy TZ, Klette KL, Wiegand R, Jemionek J, Jacobs A: Comparison of the Microgenics CEDIA heroin metabolite (6-AM) and the Roche Abuscreen ONLINE opiate immunoassays for the detection of heroin use in forensic urine samples. J Anal Toxicol 2004, 28(6):489-493.

13. Smith ML, Shimomura ET, Summers J, Paul BD, Nichols D, Shippee R, Jenkins AJ, Darwin WD, Cone EJ: Detection times and analytical performance of commercial urine opiate immunoassays following heroin administration. J Anal Toxicol 2000, 24(7):522-529.

14. Basurto FZ, Montes JMG, Cubos PF, Santed FS: Validity of the self-report on drug use by university students: Correspondence between selfreported use and use detected in urine. Psicothema 2009, 21:213-219.
15. Bush K, Kivlahan DR, MCDonell MB, Fihn SD, Bradley KA: The AUDIT alcohol consumption questions (AUDIT-C): an effective brief screening test for problem drinking. Ambulatory Care Quality Improvement Project (ACQUIP). Alcohol Use Disorders Identification Test. Arch Intern Med 1998, 158(16):1789-1795.

16. Bradley KA, Bush KR, Epler AJ, Dobie DJ, Davis TM, Sporleder JL, Maynard C, Burman ML, Kivlahan DR: Two brief alcohol-screening tests From the Alcohol Use Disorders Identification Test (AUDIT): validation in a female Veterans Affairs patient population. Arch Intern Med 2003, 163(7):821-829.

17. Chesney MA, Ickovics JR, Chambers DB, Gifford AL, Neidig J, Zwickl B, Wu AW, Patient Care Committee \& Adherence Working Group of the Outcomes Committee of the Adult AIDS Clinical Trials Group (AACTG): Self-reported adherence to antiretroviral medications among participants in HIV clinical trials: the AACTG adherence instruments. AIDS Care 2000, 12(3):255-266.

18. Peace MR, Tarnai LD, Poklis A: Performance evaluation of four on-site drug-testing devices for detection of drugs of abuse in urine. J Anal Toxicol 2000, 24(7):589-594.

19. Saitz R: Clinical practice. Unhealthy alcohol use. N Engl J Med 2005, 352(6):596-607.

20. Kroenke K, Spitzer RL, Williams JB: The PHQ-9: validity of a brief depression severity measure. J Gen Intern Med 2001, 16(9):606-613.

21. Landis JR, Koch GG: The measurement of observer agreement for categorical data. Biometrics 1977, 33(1):159-174.

22. Chander G, Himelhoch S, Fleishman JA, Hellinger J, Gaist P, Moore RD, Gebo KA: HAART receipt and viral suppression among HIV-infected patients with co-occurring mental illness and illicit drug use. AIDS Care 2009, 21(5):655-663.

23. Colon HM, Perez CM, Melendez M, Marrero E, Ortiz AP, Suarez E: The validity of drug use responses in a household survey in Puerto Rico: comparison of survey responses with urinalysis. Addict Behav 2010, 35(7):667-672.

24. Fendrich M, Mackesy-Amiti ME, Johnson TP: Validity of self-reported substance use in men who have sex with men: comparisons with a general population sample. Ann Epidemiol 2008, 18(10):752-759.

25. van Griensven F, Naorat S, Kilmarx PH, Jeeyapant S, Manopaiboon C, Chaikummao S, Jenkins RA, Uthaivoravit W, Wasinrapee P, Mock PA, Tappero JW: Palmtop-assisted self-interviewing for the collection of sensitive behavioral data: randomized trial with drug use urine testing Am J Epidemiol 2006, 163(3):271-278.

26. Kerr T, Marshall BD, Milloy MJ, Zhang R, Guillemi S, Montaner JS, Wood E: Patterns of heroin and cocaine injection and plasma HIV-1 RNA suppression among a long-term cohort of injection drug users. Drug Alcohol Depend 2012, 124(1-2):108-112.

27. Hahn JA, Samet JH: Alcohol and HIV disease progression: weighing the evidence. Curr HIV/AIDS Rep 2010, 7(4):226-233.

28. Drainoni ML, Farrell C, Sorensen-Alawad A, Palmisano JN, Chaisson C, Walley AY: Patient perspectives of an integrated program of medical care and substance use treatment. AIDS Patient Care STDS 2014, 28(2):71-81.

29. Westergaard RP, Ambrose BK, Mehta SH, Kirk GD: Provider and clinic-level correlates of deferring antiretroviral therapy for people who inject drugs: a survey of North American HIV providers. J Int AIDS Soc 2012, 15(1):10

30. SAMHSA: Center for Substance Abuse Treatment. Substance Abuse: Clinical Issues in Intensive Outpatient Treatment. Rockville (MD): Substance Abuse and Mental Health Services Administration (US). (Treatment Improvement Protocol (TIP) Series, No. 47) Appendix B. Urine Collection and Testing Procedures and Alternative Methods for Monitoring Drug Use. 2006, Available from: http://www.ncbi.nlm.nih.gov/books/NBK64092/.

31. Silverberg MJ, Ray GT, Saunders K, Rutter CM, Campbell Cl, Merrill JO, Sullivan MD, Banta-Green CJ, Von Korff M, Weisner C: Prescription longterm opioid use in HIV-infected patients. Clin J Pain 2012, 28(1):39-46.

32. McMahon JH, Manoharan A, Wanke CA, Mammen S, Jose H, Malini T, Kadavanu T, Jordan MR, Elliott JH, Lewin SR, Mathai D: Pharmacy and self-report adherence measures to predict virological outcomes for patients on free antiretroviral therapy in Tamil Nadu, India. AIDS Behav 2013, 17(6):2253-2259.

33. Liu H, Golin CE, Miller LG, Hays RD, Beck CK, Sanandaji S, Christian J, Maldonado T, Duran D, Kaplan AH, Wenger NS: A comparison study of multiple measures of adherence to HIV protease inhibitors. Ann Intern Med 2001, 134(10):968-977.

doi:10.1186/1471-2334-14-508

Cite this article as: Qian et al.: Current drug use and lack of HIV virologic suppression: point-of-care urine drug screen versus self-report. BMC Infectious Diseases 2014 14:508. 